Aim of the study: There are at least four main signal transduction pathways within human cells which are activated by interaction of an extracellular ligand with its corresponding receptors. One of them is activation of protein kinase. Actually, any of these proteins at any level of the signaling cascade in a human cell can undergo mutation and cause irregular cellular proliferation and finally result in cancer. C-kit is alternatively called stem cell factor receptor (SCFR) or CD117. It appears that lack of c-kit expression accompanies progression of some tumors, e.g. lung, breast, GIST. The aim of this study was to evaluate C-kit protein expression level within cancer cases.

Material and methods: Sixty specimens of breast cancer and 60 non-cancerous breast tissue specimens were evaluated by IHC for C-kit presentation. We used positive GIST slides as controls. Epi-info ver 6.04 (CDC, WHO) was used for analysis.

Results: C-kit was negative in all breast cancer specimens. C-kit was negative in $47(78 \%)$ of 60 non-cancerous breast tissue specimens, but was positive in $13(22 \%)$ of them $(p<0.0001)$.

Conclusions: There is a reduction in C-kit expression with malignant transformation of breast epithelium. C-kit is believed to play a role in breast carcinogenesis. However, we should follow patients with normal or benign breast tissue to indicate any correlation between C-kit presentation and breast cancer development.

Key words: C-kit protein, breast cancer, imatinib, ductal carcinoma.

\section{Expression of c-kit protein in cancer vs. normal breast tissue}

\section{Abdolhassan Talaiezadeh ${ }^{1}$, Seyed Nematollah Jazayeri ${ }^{2}$, Jamal Nateghi ${ }^{3}$}

1Department of Surgery, Imam Khomeini Hospital, Ahvaz Jundishapur University of Medical Sciences, Ahvaz, Iran

${ }^{2}$ Ahvaz Jundishapur University of Medical Sciences, Ahvaz, Iran

${ }^{3}$ Hematologist \& Oncologist, Iran

\section{Introduction}

The majority of genes that undergo mutation in human cancers have a direct role in the cell cycle, but most of them participate in signal transduction. Signal transduction pathways express proteins that regulate the cell cycle, but they do not directly participate in controlling cell cycle events. These pathways involve biochemical mechanisms through which the cell can communicate with its extracellular environment. Whether the cell receives the signal from a soluble protein or a direct cell-cell interaction, the result will be activation of a single signal transduction pathway. Subsequently increased or decreased protein synthesis resulting in a phenotype change (e.g. proliferation) will ensue. There are at least four main signal transduction pathways within human cells which are activated by interaction of an extracellular ligand with its corresponding receptors. One of them is activation of protein kinase which acts through phosphorylation of other proteins in their tyrosine (i.e. tyrosine kinases) or serine threonine (i.e. serine or threonine kinases) roots or both (i.e. dual specificity kinases). These signal transduction pathway are essential ways through which cells communicate with their environment and have a pivotal role in regulation of cellular proliferation and death [1].

These pathways consist of varying and numerous proteins in different levels of the signaling cascade. Actually, any of these proteins at any level of the signaling cascade in a human cell can undergo mutation and cause irregular cellular proliferation. As an instance, growth factor receptors, including proteins of the epidermal growth factor receptor (EGFR) family, generally undergo some changes in certain types of cancer (e.g. breast cancer). In breast cancer, growth factor receptor Her-2/neu is amplified in $40 \%$ of cases (numerous copies of the gene encoding its protein). Such gene amplification causes overexpression of Her-2/neu protein. This amplification corresponds to prognosis of the breast cancer and in vitro proliferation. One of the most important signal transduction pathways that is genetically changed in human malignancies is a protein kinase pathway called MAPK (mitogen-activated protein kinase). MAPK is probably the most well-identified signal transduction pathway and is considered as a model to understand inward transduction of an extracellular signal and its amplification within the intracellular environment. When a growth factor is bound to its receptor, the receptor undergoes a spatial change or a tyrosine phosphorylation or both. This causes activation of a protein called Grb2 which in turn activates an adaptor protein called rasGrb2/SOS. ras is a $G$ protein monomer (i.e. a guanine nucleotide binding protein) that changes GTP into GDP and subsequently is able to transmit signals to cytoplasmic proteins. Within each step, the signal is amplified and finally transcription factors are activated and gene transcription causes expression of proteins that regulate proliferation. One of the proteins that are upregulated by the MAPK pathway is cyclin D, which facilitates entering G1 from G0 and passing from G1 into S phase. Therefore, an extracellular signal that activates a growth factor receptor may 
cause increased proliferation through increased expression of cyclin D. It has been indicated that the majority of genes encoding MAPK pathway proteins are mutated in human malignancies and, theoretically, mutation of each protein in this pathway can cause increased proliferation as in colorectal carcinoma [2]. In addition, most of the genes in the MAPK pathway are ras proteins that undergo mutation in numerous malignancies including 95\% of pancreas adenocarcinoma cases [3].

C-kit is alternatively called stem cell factor receptor (SCFR) or CD117. Its corresponding gene is located on chromosome $4 \mathrm{q} 12$. The DNA for this gene is $89 \mathrm{~kb}$ long and contains 21 exons. Following transcription, mRNAs are produced that are $5.23 \mathrm{~kb}$ long and after splicing of number 9 alternative exon, two isoforms called kit and kitA are produced. The difference between these two is in the presence or absence of four amino acids. The resultant protein is a type III tyrosine kinase receptor that contains 976 amino acids and weighs $145 \mathrm{kDa}$. This receptor contains an extracellular arm with five Ig-like rings, one extremely hydrophobic transmembrane arm (containing 23 amino acids) and an intracellular arm with tyrosine kinase function. This protein receptor is presented and expressed on hematopoietic stem cells, mast cells, melanocytes and germ-cell lineage cells [4]. This protein receptor is located on plasma membrane [4]. Binding of its corresponding ligand, i.e. stem cell factor, causes receptor dimerization, its auto-phosphorylation and eventually signal transduction through molecules containing $\mathrm{SH}-2$ arms. It appears that lack of c-kit expression accompanies progression of some tumors (e.g. melanoma) and autocrine as well as paracrine stimulation and the c-kit/SCF system may play a role in human solid tumors such as malignancies of lung, breast, testis and gynecologic dysplasias [5].

Therefore, as described above, C-kit protein is a kinase necessary for cell division (i.e. in hematopoietic cells), showing decreased or increased expression in some malignancies [6]. It is seen with increasing expression in different stromal tumors, especially in the gastrointestinal tract, and is directly associated with tumor growth [7]. Inhibition of this protein can help prevent tumor growth especially in metastatic cases by counteraction of a monoclonal antibody called imatinib marketed as Glivec ${ }^{\circledR}$, which was initially manufactured by Novartis company.

The aim of this study was to evaluate C-kit protein expression level within the very common breast cancer cases.

\section{Material and methods}

Sixty tissue blocks of breast cancer tissues and 60 noncancerous breast tissue blocks were collected from pathol- ogy department archives. Hematoxylin and eosin sections of all specimens were reviewed to confirm the tumor type and histology. All cases were immunostained for C-kit, product of Novocastra company UK (clone No. T595).

For immunohistochemical (IHC) analysis of the C-kit protein, sections were dewaxed and dehydrated, and antigen retrieval was performed by microwave heating for 15 minutes in a $10 \mathrm{mM}$ citrate buffer at $\mathrm{pH}$ 6. Then, the sections were reacted with rabbit polyclonal antibody for C-kit. The specimens were diluted at room temperature, and then were subsequently stained by the universal immuno- peroxidase polymer method using a Histofine Simple Stain MAX PO(M) kit, according to the protocol provided by the manufacturer. Positive reactions were visualized with diaminobenzidine, followed by counterstaining with hematoxylin. Due to the lack of an internal control in breast tissue, we used C-kit positive GIST slides as the control in our study. $\chi^{2}$ was used for comparison.

\section{Results}

The immunohistochemical expression of the C-kit protein was determined to be negative in all breast cancer specimens. Of 60 cases with malignancy, the histological type of breast cancer in 53 (88.33\%) was ductal carcinoma, 5 (8.33\%) cases were ductal carcinoma in situ (DCIS), 1 (1.67\%) case was medullary carcinoma and 1 (1.67\%) case was lobular carcinoma.

Of 60 cases, C-kit was negative in all cases. Of 60 controls, C-kit was positive in $13(21.66 \%)$ cases $(p<0.001)$.

Of 60 controls, the C-kit expression was negative in 47 (78\%) specimens, and positive in 13 (22\%) subjects (Table 1 ).

The histological types of non-cancerous breast tissue in patients with positive C-kit expression were 6 cases of fibrocystic changes (Fig. 1), 3 cases of fibro-adenoma (Fig. 2) and 4 cases of normal breast tissue (Fig. 3).

\section{Discussion}

C-kit protein, a 145 kDa tyrosine kinase (CD117) with oncogenic properties, is a transmembrane growth factor receptor known as a stem cell factor (SCF). It is encoded by the $\mathrm{C}$-kit proto-oncogene located on chromosome 4q11-q12. Activation of C-kit by its SCF ligand leads to dimerization of the receptor. The latter activates further signaling cascades that control cell proliferation, adhesion and differentiation. Ulivi et al. showed such expression in normal and tumor breast tissue [8]. Several studies have identified the presence of a C-kit malignant mutation in over half of gastrointestinal stromal tumors (GISTS), as well as in other human tumors, including lung cancer, germ cell tumors, neuroblastoma, melanoma, ovarian cancer and breast carcinoma [9-11].

Table 1. Distribution of C-kit among cancerous and non-cancerous specimens

\begin{tabular}{lclc} 
Cancerous & C-kit & Non-cancerous & $47(-), 4(+)$ \\
\hline ductal carcinoma (53) & - & normal (51) & + \\
ductal carcinoma in situ (5) & - & fibrocystic changes (6) & + \\
medullary carcinoma (1) & - & fibroadenoma (3) & \\
lobular carcinoma (1) & - & &
\end{tabular}




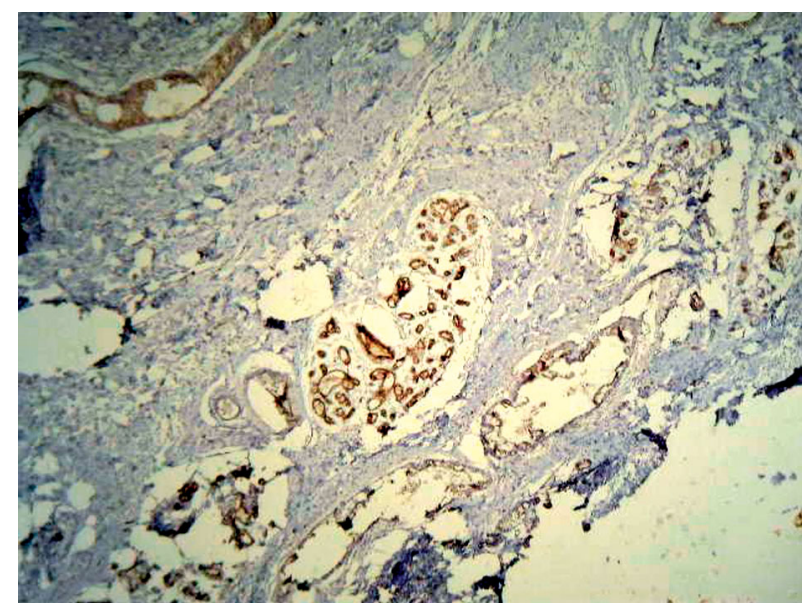

Fig. 1. Immunohistochemical expression of C-kit in fibrocystic disease in breast

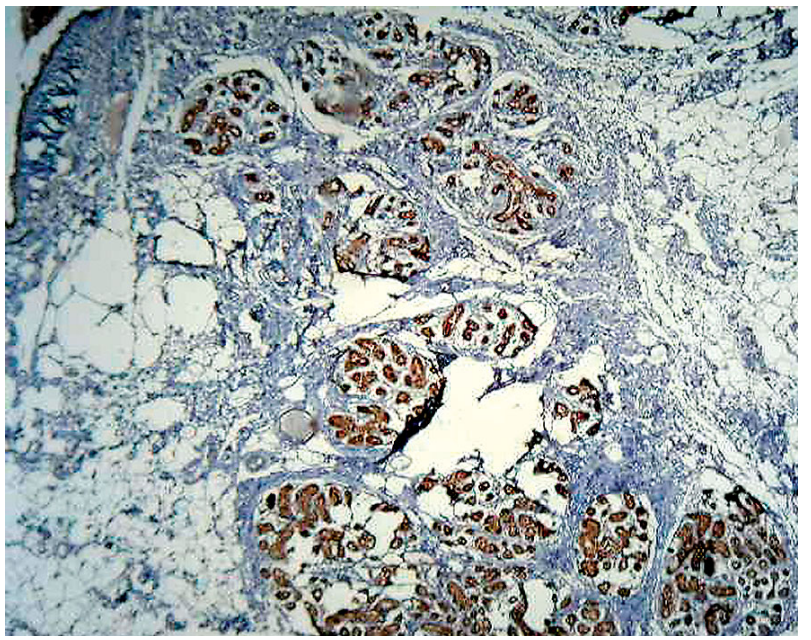

Fig. 3. Immunohistochemical expression of C-kit in normal breast tissue

Interestingly, over-expression of C-kit has been found to affect proliferation in human neural, lung, breast, colorectal, skin and prostatic tumors [12-14]. Although the functional role of mutated C-kit kinase activity is not fully understood, it seems that in breast, thyroid and ovarian cancer, the malignant transformation correlates with loss of C-kit protein expression. Immunohistochemical expression of C-kit protein is a rare event in poorly differentiated carcinoma [15].

C-kit is also physiologically expressed in hematopoietic stem cells, tissue stem cells, tissue mast cells, germ cells, melanocytes, interstitial cells of Cajal and mammary gland epithelium, as we showed its expression in normal breast tissues. However, C-kit is not usually expressed in normal squamous epithelium or the glandular epithelium of the lung, endocervix, pancreas, prostate, stomach or the small and large intestines $[16,17]$.

The diverse expression of C-kit in normal tissue results in the diverse expression of C-kit in tumor tissue originating from such normal tissues. Such opposite and polar effects presented by Diallo et al. underline that C-kit expression rep-

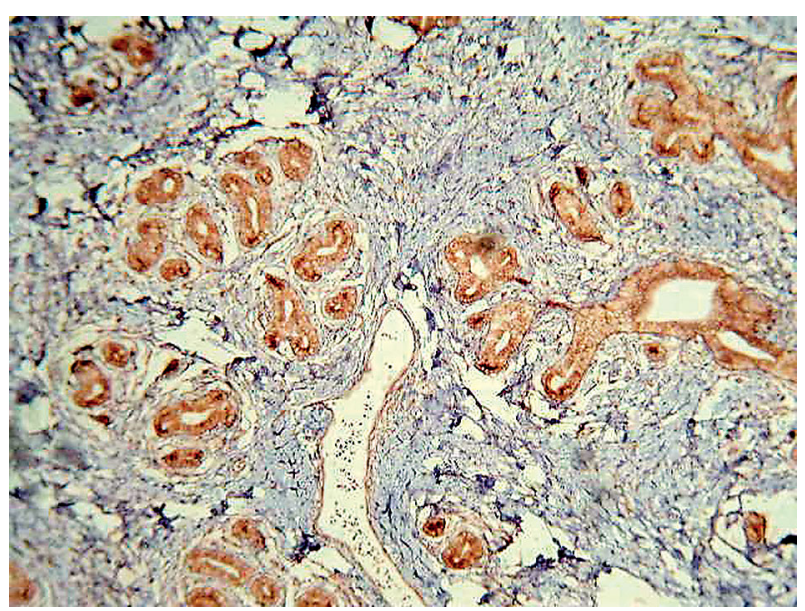

Fig. 2. Immunohistochemical expression of C-kit in breast fibroadenoma

resents an independent negative prognostic marker in high-risk breast cancer, and the findings of Ulivi et al. suggest that C-kit/SCF plays an important role in the maintenance of normal growth of mammary epithelium and that the process of malignant transformation is accompanied by their progressive loss $[8,18]$. Therefore, the aim of the present study was to evaluate the C-kit expression of breast cancer in comparison with normal breast tissue.

C-kit is physiologically expressed in the normal gland of breast tissue [19]. However, other findings indicate that C-kit is involved in growth and maintenance of the normal epithelium and the C-kit function may be lost following malignant transformation [20]. The rate of positive C-kit expression in breast cancer varies from 1 to $82 \%$, which is likely attributable to the different methods for determination of C-kit expression. The rate of positive C-kit expression in normal breast tissue was 100\% in four studies [21]. Although the rate of positive C-kit expression varied in the different studies, the rate of positive C-kit expression of benign tumor was always lower than that of normal breast tissue and the rate of positive C-kit expression of breast cancer was always lower than that of benign tumors in each study [22].

As there is a reduction in C-kit expression with malignant transformation of breast epithelium, C-kit is believed to play a role in breast carcinogenesis. The Chang et al. research result suggests that the loss of expression of this protein might correlate with malignant breast cancer progression, but it is most likely involved at an early stage of human breast cancer development [23].

In our study we should follow patients with normal or benign breast tissue to indicate any correlation between C-kit presentation and breast cancer development. However, we cannot say that any positive or negative effect of C-kit expression on breast cancer was proved by this study.

\section{References}

1. Cantley L, Carpenter CL. Cell signaling. In: DeVita, Vincent T, Lawrence TS, Theodore S.; Rosenberg SA, Steven A. DeVita, Hellman \& Rosenberg's Cancer: Principles \& Practice of Oncology. Lippincott Williams \& Wilkins, Philadelphia 2008; 67-77. 
2. Bellone G, Silvestri S, Artusio E, et al. Growth stimulation of colorectal carcinoma cells via the C-Kit receptor is inhibited by TGF-beta 1. J Cell Physiol 1997; 172: 1-11.

3. Esposito I, Kleeff J, Bischoff SC, et al. The stem cell factor-C-Kit system and mast cells in human pancrea cancer. Lab Invest 2002; 82: 1481-92.

4. Takahashi H, Saitoh K, Kishi H, Parsons PG. Immunohistochemica localisation of stem cell factor (SCF) with comparison of its receptor c-Kit proto-oncogene product (c-KIT) in melanocytic tumours. Virchows Arch 1995; 427: 283-8.

5. Nishida K, Tsukamoto T, Uchida K, Takahashi T, Takahashi T, Ueda R. Introduction of the C-Kit gene leads to growth suppression of a breast cancer cell line, MCF-7. Anticancer Res 1996; 16(6B): 3397-402.

6. Linnekin D. Early signaling pathways activated by c-Kit in hematopoietic cells. Int J Biochem Cell Biol 1999; 31: 1053-74.

7. Fletcher CD, Berman JJ, Corless C, Gorstein F, Lasota J, Longley BJ. Diagnosis of gastrointestinal stromal tumors: a consensus approach. Hum Pathol 2003; 33: 459-65.

8. Ulivi P, Zoli W, Medri L, Amadori D, Saragoni L, Barbanti F, Calistri D, Silvestrini R. C-kit and SCF expression in normal and tumor breast tissue. Breast Cancer Res Treat 2004; 83: 33-42.

9. Hirota S, Isozaki K, Moriyama Y, et al. Gain-of-function mutations of c-kit in human gastrointestinal stromal tumors. Science 1998; 279: 577-80

0. Beck D, Gross N, Brognara CB, Perruisseau G. Expression of stem cell factor and its receptor by human neuroblastoma cells and tumor. Blood 1995; 86: 3132-8.

11. Dipoala RS, Kuczynski WI, Onodera K, Retajczak MZ, Hijiya N, Moore J. Evidence of a functional kit receptor in melanoma, breast and lung carcinoma cells. Cancer Gene Ther 1997; 4: 176-82.

12. Sammarco I, Capurso G, Coppola L, et al. Expression of the protooncogene C-kit in normal and tumor tissue from colorectal catci noma patients. Int J Colorectal Dis 2004; 19: 545-53.

13. Simak R, Capodieci P, Cohen DW, et al. Expression of C-kit and kitligand in benign and malignant prostatic tissue. Histol Histopathol 2000; 15: 365-74

14. Hibi K, Takahashi T, Sekido Y, Ueda R, Hida T, Ariyoshi Y, Takagi H, Takahashi T. Coexpression of the stem cell factor and the c-kit genes in small-cell lung cancer. Oncogene 1991; 6: 2291-6.

15. Reed J, Ouban A, Schickor FK, Murarca P, Yeatman T, Coppola D. Immunohistochemical staining for C-kit(CD117) is a rare event in human colorectal carcinoma. Clin Colorectal Cancer 2002; 2: 119-22.

16. Vanucchi MG. receptors in iterstitial cells of Cajal: identification and possible physiology roles. Microsc Res Tech 1999; 47: 325-35.

17. Turner AM, Zsebo KM, Martin F, Jacobsen FW, Bennett LG, Broody VC. Nonhematopoietic tumor cell in lines express stem cell factor and display C-kit receptors. Blood 1992; 80: 374-81.

18. Diallo R, Ting E, Gulz O, et al. C-kit expression in high-risk breast cancer subgroup treated with high-dose or conventional dose-dense chemotherapy. Verh Dtsch Ges Pathol 2006; 90: 177-85.

19. Polat A. C-kit expression in columnar cell lesions of breast accom panied by benign and malignant breast diseases. Pathol Res Pract 2007; 203: 765-9.

20. Tsutsui S, Yasuda K, Suzuki K, Takeuchi H, Nishizaki T, Higashi H, Era S. A loss of C-kit expression is associated with an advanced stage and poor prognosis in breast cancer. Br J Cancer 2006; 94: 1874-8

21. Yared MA, Middleton LP, Meric F, Cristofanilli M, Sahin AA. Expression of c-kit proto-oncogene product in breast tissue Breast J 2004; 10: 323-7.

22. Tsuura Y, Suzuki T, Honma K, Sano M. Expression of C-kit protein in proliferative lesions of human breast: sexual defference and close association with phosphotyrosine status. J Cancer Res Clin Oncol 2002; 128: 239-46.

23. Ko CD, Kim JS, Ko BG, et al. The meaning of the C-kit proto-oncogene product in malignant transformation in human mammary epithelium. Clin Exp Metastasis 2003; 20: 593-7.

\section{Address for correspondence}

Abdolhassan Talaiezadeh

Department of Surgery

Imam Khomeini Hospital

Ahvaz Jundishapur University of Medical Sciences

Ahvaz, Iran

e-mails: ahtalaiezadeh@yahoo.com;

Ah.talaiezadeh@ajums.ac.ir

\section{Submitted: $\quad 6.06 .2011$ \\ Accepted: $\quad 15.06 .2012$}

